\title{
Investigation of Smallest Volume Difference Measurable Using 3D Imaging
}

\author{
Zhale NOWROOZILARKI ${ }^{1}$, Mary Catherine BORDES ${ }^{2}$, Urmila SAMPATHKUMAR ${ }^{3}$, \\ Summer E. HANSON ${ }^{2}$, Gregory P. REECE ${ }^{2}$, Mia K. MARKEY ${ }^{4,5}$, Fatima A. MERCHANT ${ }^{1,3,4}$ \\ ${ }^{1}$ Department of Engineering Technology, University of Houston, Houston, TX, USA; \\ ${ }^{2}$ Dep. of Plastic Surgery, The University of Texas MD Anderson Cancer Center, Houston, TX, USA; \\ ${ }^{3}$ Department of Computer Science, University of Houston, Houston, TX, USA; \\ ${ }^{4}$ Department of Biomedical Engineering, The University of Texas at Austin, Austin, TX, USA; \\ ${ }^{5}$ Dep. of Imaging Physics, The University of Texas MD Anderson Cancer Center, Houston, TX, USA
}

https://doi.org/10.15221/20.26

\begin{abstract}
With the advent of three-dimensional (3D) surface imaging technology, objective measurements of breast aesthetics, such as contour, surface area, shape, size, and volume are now possible, and studies have demonstrated its utility and efficacy in clinical medicine [1]. Moreover, several relatively low-cost portable hand-held 3D scanners are now commercially available, which has increased the likelihood of the technology finding widespread clinical adoption. An understanding of the capabilities and limitations of 3D imaging technology and corresponding measurements is essential for its successful application in pre-operative planning as well as post-operative assessments. Breast volume is a key outcome measure in aesthetic and reconstructive surgery; thus, it is critical to not only have validated algorithms for accurate measurements, but to also understand the ability of the technology to resolve and detect volume changes. Inconsistencies in volume measurement and lack of appropriate standardization protocols can influence applications that utilize quantitative measurements. For example, changes in breast volume need to be quantified to maintain breast symmetry and any inconsistencies may impact the surgeon's ability to formalize pre-operative plans. In this study, we assessed the resolution of 3D imaging in terms of assessing breast volume change and compared volume measurements made using a non-portable 3D imaging system (3dMDTorso ${ }^{\mathrm{TM}}$ ) and a hand-held 3D scanner (Go!SCAN 3D ${ }^{\mathrm{TM}}$ ). Using adjustable implants with varying volumes, with the non-portable system and customized volume computation software, we could reliably detect changes in volume as low as $5.5 \pm 2.6 \mathrm{cc}$. In addition, volume measurements made using the non-portable system and the hand-held scanner were highly correlated $\left(R^{2}=0.99\right)$, demonstrating feasibility of using the portable hand-held scanner for breast volume measurements.
\end{abstract}

Keywords: Breast volume, Volume measurement accuracy, 3D surface imaging

\section{Introduction}

Breast volume is one of the key metrics used for assessing breast surgery outcomes. There are different techniques to measure the breast volume such as anthropometry, thermoplastic casting, the Archimedes procedure, the Grossman-Roudner device, mammography, computed tomography, ultrasonography, Magnetic Resonance Imaging (MRI) and 3D surface imaging. While the water displacement approach is inexpensive and straightforward in principle, in practice it is cumbersome and time-consuming. Also, it is not feasible to use this approach for many patients during post-operative stages. MRI provides fairly accurate measurements (error of $<10 \%$ ) and is considered the gold standard for assessing breast volume, but the procedure is time-consuming and expensive, which limits its use for routine pre-operative planning and post-operative assessment [2]. In contrast, 3D surface imaging provides several advantages for routine use, being non-invasive, fast, relatively inexpensive and convenient both to the patient and to the technician. Also, the patient can be imaged in the standing position which is a typical torso position in everyday life, as opposed to the prone positioning of MRI. In breast surgery, volume measurements are largely used in determining breast symmetry and aesthetic outcomes. In order to assess the limits of 3D imaging technology in terms of detecting breast volume changes, we investigated volume measurements in images captured using the 3dMDtorso ${ }^{\mathrm{TM}}$ system (3dMD ${ }^{\mathrm{TM}}$ LLC, Atlanta, GA) and computed using customized software developed by our research group [3]. We also assessed the Go!SCAN 3D ${ }^{\mathrm{TM}}$ (Creaform Inc., Canada) hand-held scanner for enabling volume measurements in breast surgery. 


\section{Previous work}

Several methods have been utilized for breast volume measurements. Kayer et al., 2011 utilized five different methods, including mammography, anatomical measurements (anthropometric), thermoplastic casting, the Archimedes method and the Grossman-Roudner device, to measure the breast volume of 30 patients who were scheduled to undergo mastectomy. The volume of the removed mastectomy specimen was measured using the water displacement method as the gold standard volume. The authors then compared the specimen volume to the volume which was measured using the five different methods. Statistical analysis demonstrated reliability (r-values) of 0.997, 0.975, 0.934, 0.989 and 0.94 for the mammographic, anthropometric, Grossman-Rounder, Archimedes, and thermoplastic casting methods, respectively [4]. However, it should be noted that the volume of the mastectomy specimen is not precisely equivalent to the volume of the pre-operative breast, as it does not include the skin or subcutaneous fat. In another study, Howes et al., 2017 compared 3D laser scanning technology against MRI imaging as the gold standard for breast volume measurement [5]. They used two different statistical methods, Pearson correlation and Bland-Altman to demonstrate a strong association between MRI and 3D imaging. Faith Hyun Kyung et al., 2020 has proposed the following approach to measure the differential volume using subtraction of two captured surfaces in order to address challenges encountered when computing breast volume using surface imaging, including issues related to (1) determining breast boundary (especially along the inferior positions of the breast, as seen with occlusion of the inframammary fold in case of high ptosis grades) and (2) prediction of the chest wall. Using the $3 \mathrm{dMDtorso} 0^{\mathrm{TM}}$, they imaged each patient twice, first with the subject positioned upright at $90^{\circ}$ and then posteriorly inclined at $30^{\circ}$. The breast volume at each angle was measured using $3 \mathrm{dMDvultus}{ }^{\mathrm{TM}}$ software. They found a statistically significant difference between the actual volume and the volume at $90^{\circ}$, but no difference between the actual volume and the volume at $30^{\circ}$. However, there was good correlation between the actual volume and the volume computed from surface images at both angles, with $r=0.77$ and $r=0.85$ for $90^{\circ}$ and $30^{\circ}$, respectively. They recommend positioning the subject at $30^{\circ}$ for determination of breast volume because it provides better exposure of the inferior breast [6].

Several studies have also validated commercially available 3D imaging systems for use in breast surgery. O'Connell et al., 2018 validated the Vectra XT TM imaging system for measuring breast volume and breast volume symmetry following breast reconstruction[7]. They created phantom models made of plasticine (NCP Newclay Products, Devon, UK) to simulate 6 different breasts sizes, with volumes $100 \mathrm{cc}, 200 \mathrm{cc}, 300 \mathrm{cc}, 400 \mathrm{cc}, 500 \mathrm{cc}$ and $1000 \mathrm{cc}$. The phantoms were placed on a wooden board held 1.5 meters from the imaging system and the region of interest around the perimeter of the phantom that includes an area $2 \mathrm{~cm}$ beyond the boundary of the model was identified. Furthermore, they performed 5 sequential resections to the phantoms within the volume range of $100 \mathrm{cc}-400 \mathrm{cc}, 9$ resections to the phantom with $500 \mathrm{cc}$ volume, and 10 resections to the phantom with $1000 \mathrm{cc}$ volume. The gold standard volume was measured using water-displacement method initially and after each resection. Two observers measured the volume of the phantoms from the images. The volume measurement accuracy was then measured by comparing the mean volumes of 10 different measurements of each observer for each phantom measured by 3D imaging to the gold standard using the following equation ((mean 3D surface image volume - gold standard volume)/gold standard $\times 100$ ). In terms of the accuracy, the authors reported 2.17 and $2.28 \%$ of mean relative differences compared to the gold standard for observer 1 and 2 respectively.

Wesselius et al., 2018 compared the accuracy of three software applications; 3D BreAST ${ }^{\mathrm{TM}}, 3 \mathrm{dMD}$ Vultus $^{\text {TM }}$, and VECTRA ${ }^{\mathrm{TM}}$, for breast volume calculations. 3D surface images were taken by VECTRA$\mathrm{XT}^{\mathrm{TM}}$ stereophotogrammetry device and breast volume was computed using the 3 different software packages. The volume of excised mastectomy specimen was estimated by considering $1 \mathrm{gr}$ of breast tissue is equivalent to $1 \mathrm{ml}$ and was used as the ground truth for comparisons. However, as mentioned before, the volume of the mastectomy specimen is not equivalent to the breast volume. The 3D BreAST $^{\mathrm{TM}}$, 3dMD Vultus ${ }^{\mathrm{TM}}$, and VECTRA ${ }^{\mathrm{TM}}$ applications provided mean volume calculation errors of 21,186 , and $-32 \mathrm{ml}(p=0.27, p<0.001$, and $p=0.14)$ or $2 \pm 25,48 \pm 26$, and $-6 \pm 27$ percent of the breast volume $(p=0.67, p<0.001$, and $p=0.16)$, respectively [8]. In terms of portable devices, Henseler et al., 2013 used a Kinect Recording System by Microsoft Corporation for 3D breast assessment [9]. They imaged nine silicone breast implants with known volumes. Furthermore, they validated the device by reporting an error margin of $10 \%$ and concluded that the reproducibility of measurements was satisfactory. Their report of a $10 \%$ acceptable error margin was further confirmed in a systematic review on the accuracy of different breast volume measurement methods performed by Choppin et al., 2016 [10], which concluded that a $5 \%$ to $10 \%$ measurement error appears to be acceptable for decision making in clinical practice. However, there is a scarcity in studies reporting the change in volume 
detectable using the different imaging methods. In this study, we investigate the amount of volume change detectable using the $3 \mathrm{dMDtorso}^{\mathrm{TM}}$ in conjunction with customized software for volume measurement. In addition, we also report on the feasibility of using a commercially available hand-held scanner; Go!SCAN 3D ${ }^{\mathrm{TM}}$, for volume measurements.

\section{Methods and materials}

\subsection{Image Acquisition}

3D surface images were acquired at The University of Texas MD Anderson Cancer Center (Houston, TX), using two different devices: the 3dMDtorso ${ }^{\mathrm{TM}}$ system (3dMD ${ }^{\circledR}$ LLC, Atlanta, GA) and Go!SCAN $3 \mathrm{D}^{\mathrm{TM}}$ hand-held scanner (Creaform Inc., Canada). The 3dMDtorso ${ }^{\mathrm{TM}}$ system is equipped with six modular cameras which use stereophotogrammetry to estimate a 3D surface image from pairs of 2D photographs. A schematic of the customized 3dMDtorso ${ }^{T M}$ and a picture of the Go! SCAN3D ${ }^{T M}$ in use at MD Anderson Cancer Center is shown in the Fig. 1. The Go!SCAN 3D ${ }^{\mathrm{TM}}$ portable hand-held scanner uses structured light 3D scanning technology and is equipped with the VXelements ${ }^{\mathrm{TM}}$ software that facilitates scanning. In order to capture the topology of the object, which is being scanned, the device flashes a light with a specific pattern (e.g. similar to a QR code) which is cast on the 3D object. Light cast on the object deforms based on the formation and the topology of the object. Two cameras included in the Go!SCAN 3D ${ }^{\mathrm{TM}}$ hand-held scanner registers this deformation and the software is then able to compute the 3D properties of the object. Both devices, output the 3D surface of the objects by providing the triangular mesh or the point cloud. If the texture image is also available, it can be overlaid on the triangular mesh to provide realistic displays of the imaged object. The hand-held device model used in this study does not capture the texture of the object's surface, whereas the 3dMDtorso ${ }^{\mathrm{TM}}$ system captures the texture, which is used to generate a 3D mesh with texture mapping (see Fig. 2.).

A

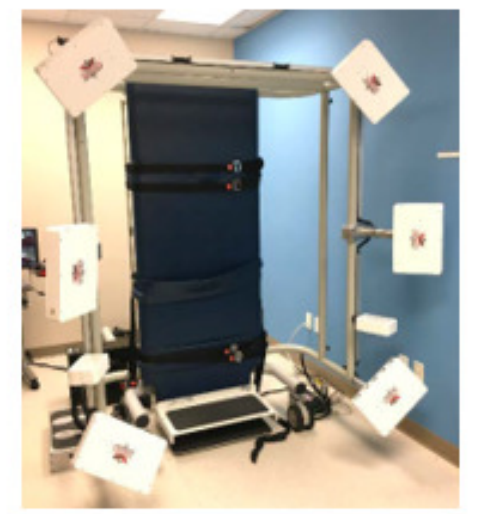

B

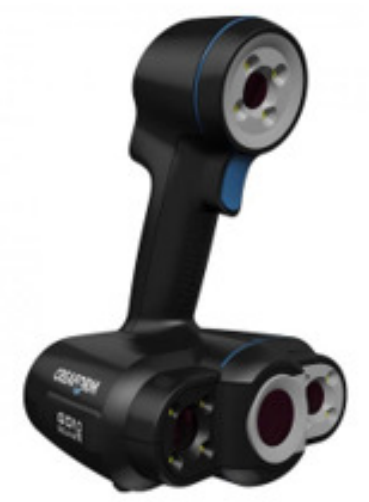

Fig. 1. Customized $3 d M D$ torso ${ }^{T M}$ imaging system at MDACC consisting of four modular camera units $(A)$. Go!SCAN 3D ${ }^{T M}$ hand-held scanner (B) [11]

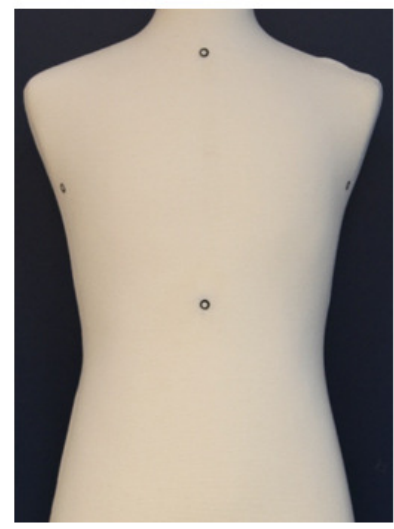

(A)

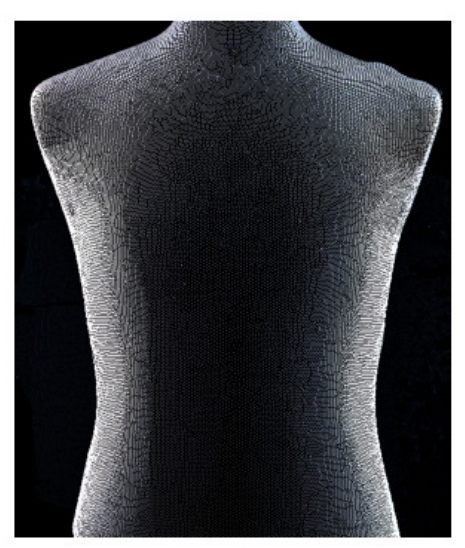

(B)

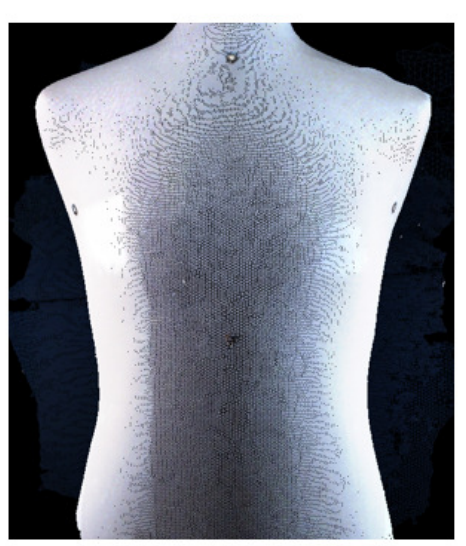

(C)

Fig. 2. Three different displays of a single 3D image of a flat chested mannequin obtained using the 3dMDtorso ${ }^{T M}$ system: A) $2 D$ texture overlaid on the $3 D$ surface mesh, B) $3 D$ point cloud, and C) $3 D$ triangular surface mesh. 


\subsection{Adjustable Implants}

In order to simulate different breast sizes in different orientations, we used saline-filled, adjustable volume, anatomically-shaped breast implants. These implants are fitted with a fill tube for allowing injection of varying amounts of water to achieve the desired size adjustments. Three different implant sizes (REF: 354-2511: 275-330 cc, REF: 354-2514: 550-660 cc, and REF: 354-2515: 650-780 cc) were donated by Mentor Worldwide LLC, Irvine, CA. The implants were painted with washable paints to minimize reflective glare and facilitate image acquisition, placed on a poster board and 3D surface images were acquired using the $3 \mathrm{dMDtorso}^{\mathrm{TM}}$ system (3dMD® LLC, Atlanta, GA).

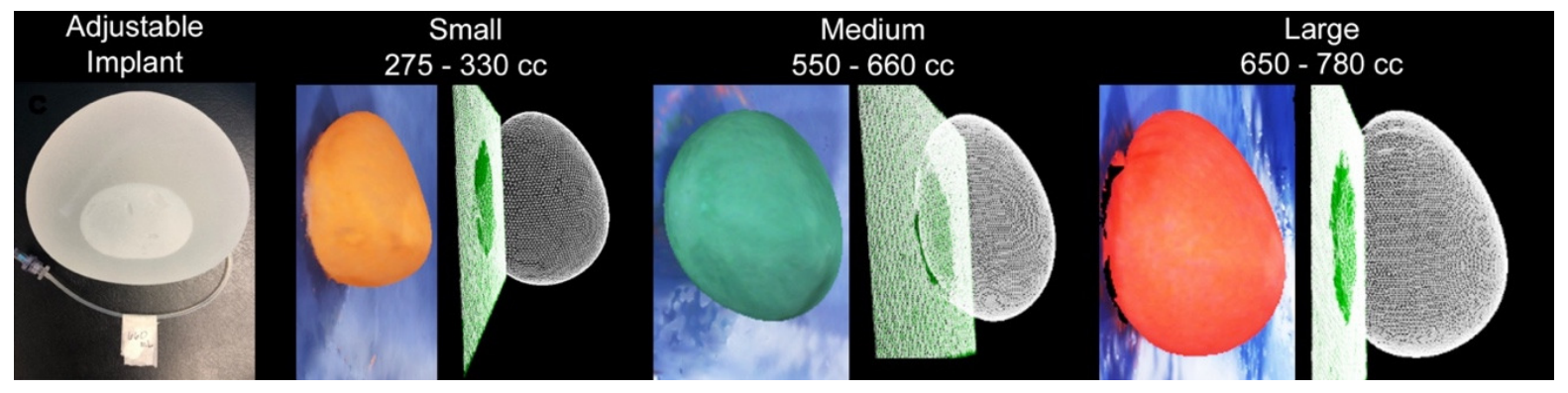

Fig. 3. Water-filled adjustable implants in three different sizes and their 3D surface mesh images.

\subsection{Phantom for Breast Volume Measurements}

In order to simulate the female torso, a flat chested mannequin (Fig. 4 (A)) was used with medium size adjustable implants (volume range of 550 - $660 \mathrm{cc}$ ), which were held in place with a body suit (HPFY Medical Supplies) as shown in Fig. 4 (B). To facilitate detection of fiducial points on the images, stickers were placed on the fiducial points manually annotated by a plastic surgeon. 3D surface images of the mannequin were obtained using the Go!SCAN 3D ${ }^{\mathrm{TM}}$ hand-held scanner and the 3dMDtorso ${ }^{\mathrm{TM}}$ system (see Fig. 5)
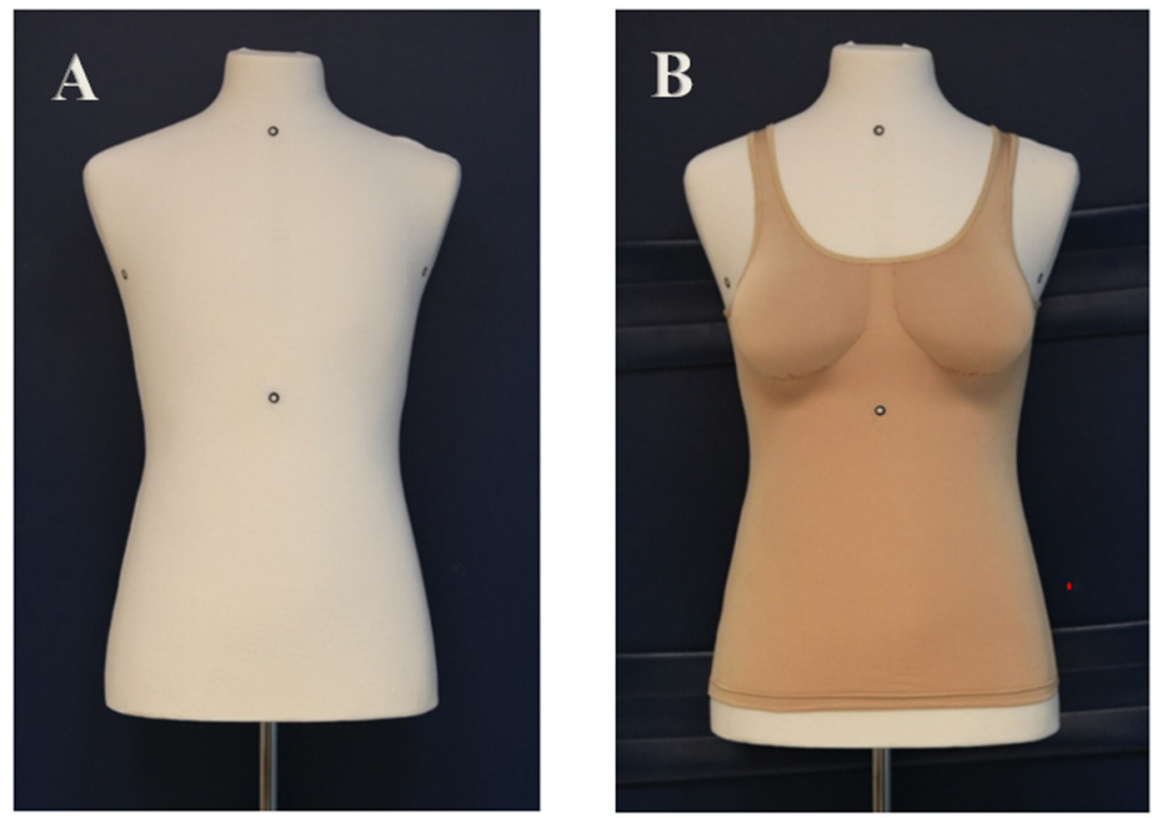

Fig. 4. Phantom for volume measurements. (A) Flat chested mannequin and $(B)$. Flat chested mannequin with implant inserts in the bodysuit. 

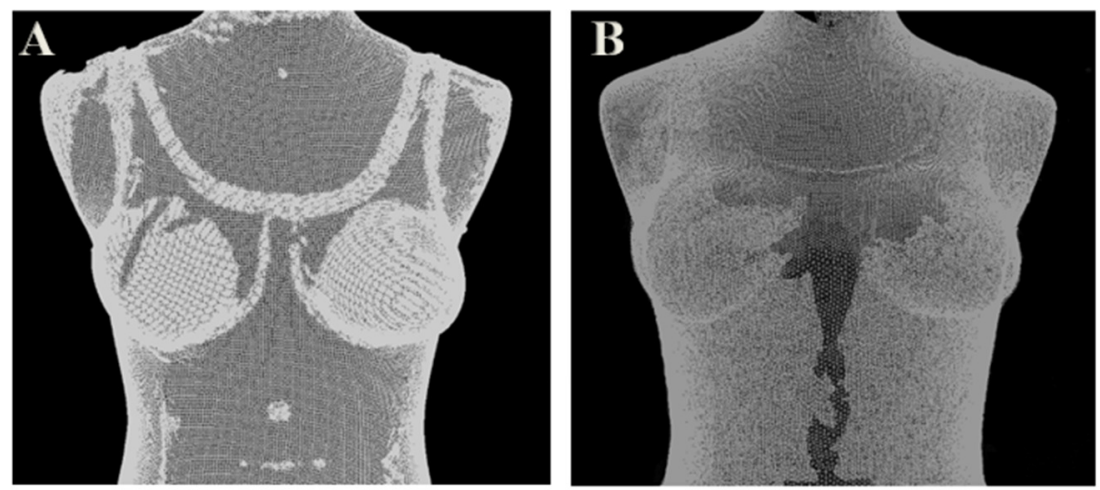

Fig. 5. 3D mesh surface of the flat-chested mannequin with the implants using (A) GO!SCAN 3D ${ }^{T M}$ hand-held scanner and (B) 3dMDtorso ${ }^{T M}$ system.

\subsection{Volume Measurement Software}

In order to measure the volume, we used a custom software developed and validated by our research team [3]. Our Java-based software facilitates interactive visualization of 3D images, annotation of fiducial points, and measurements of Euclidean as well as the contour distance between points, registration and overlay of 3D images, and volume measurements. Volume measurements were made by first delineating the surface area of the breast mound via four landmark points located at the four corners enclosing the breast (see Fig. 6). These four points define four boundary curves, which are then used to computationally estimate a parametric surface known as "Coons patch" [12]. The computationally determined Coons patch is a $3 D$ surface that parallels the surface curvature of the chest wall, thereby simulating the base of the breast. The total breast volume is then computed as the volume enclosed between the Coons patch (estimated chest wall) and the breast surface.
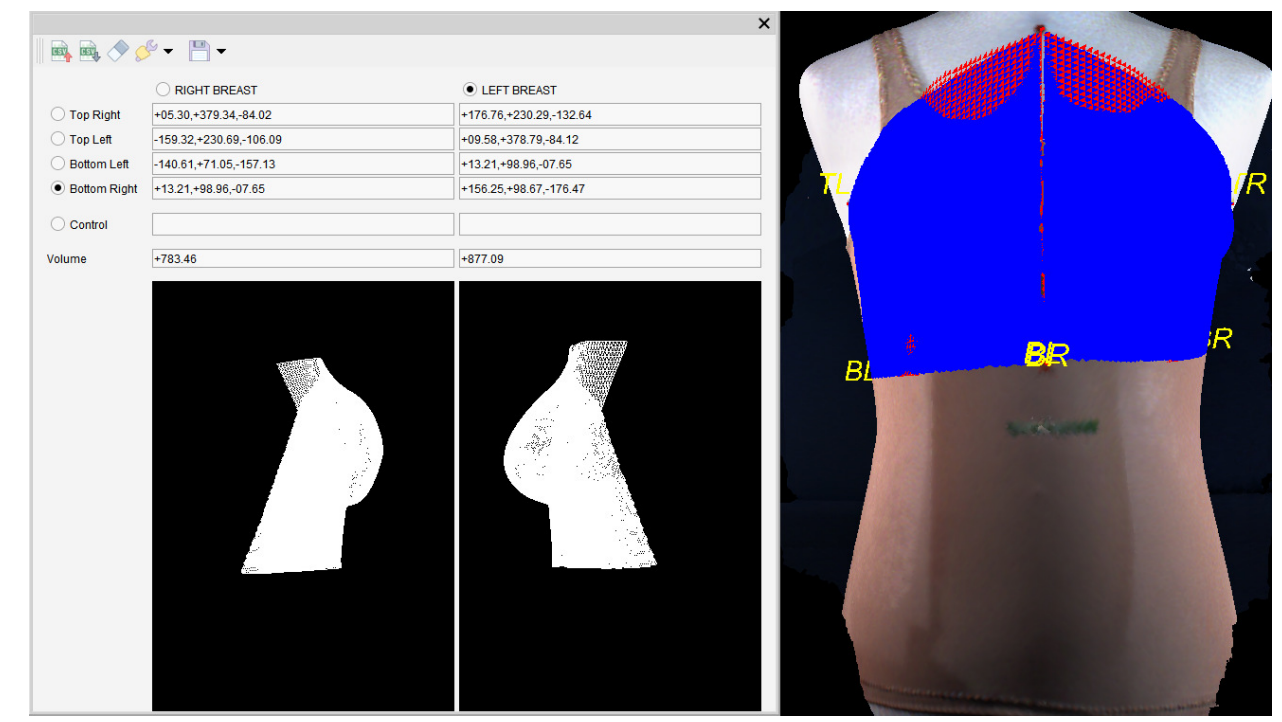

Fig. 6. Volume measurement in the customized research software. The blue mesh represents the skin surface measured whereas the red mesh represents the estimated Coons Patch, which extends behind the blue meshed area.

\section{Results}

\subsection{Comparison of 3D hand-held Scanner versus 3dMD System}

The flat chested mannequin was used with different known volumes of the medium size adjustable implant to simulate the female breasts and imaged using the GO!SCAN 3D ${ }^{\mathrm{TM}}$ hand-held scanner and the $3 \mathrm{dMDtorso} \mathrm{TM}^{\mathrm{TM}}$ system. A total of 24 measurements were made with a starting implant volume of 550 $\mathrm{cc}$ and ending volume of $660 \mathrm{cc}$, with $10 \mathrm{cc}$ increments made on each of the two sides as follows. First, the volume of the implant on the right side was incremented in $10 \mathrm{cc}$ steps up to the maximum value, and then the volume of the left side implant was similarly incremented. Implant volumes for the phantom were computed as discussed earlier (Fig. 6) using the annotated stickers to ensure consistent selection of the landmark points. The adjusted volume was measured by subtracting two terms from the 
measured volume to account for any added volume due to the bodysuit (used to hold the implant on the mannequin) and the implant material: (1) the volume of the flat-chested mannequin with the body suit, but without the implants, and (2) the volume of the empty implant, which was estimated using its density $(0.975 \mathrm{grams} / \mathrm{cc}$ ) and its mass (46 grams) (volume $=$ mass $/$ density). The absolute error, i.e., |adjusted volume - injected volume|, was measured at $33 \pm 20 \mathrm{cc}$ or $5.58 \pm 3.46 \%$ and $32 \pm 18 \mathrm{cc}$ or 5.39 $\pm 3.05 \%$ using the images obtained by the Go!SCAN 3D ${ }^{\mathrm{TM}}$ hand-held scanner and the 3dMDtorso ${ }^{\mathrm{TM}}$ system respectively. For both systems, the measurement error was less than $6 \%$.

As shown in Fig. 7 volume measurement on images from the GO!SCAN 3D ${ }^{\mathrm{TM}}$ hand-held scanner is highly correlated with the volume measurements on images taken by the $3 \mathrm{dMDtorso}^{\mathrm{TM}}$ system.

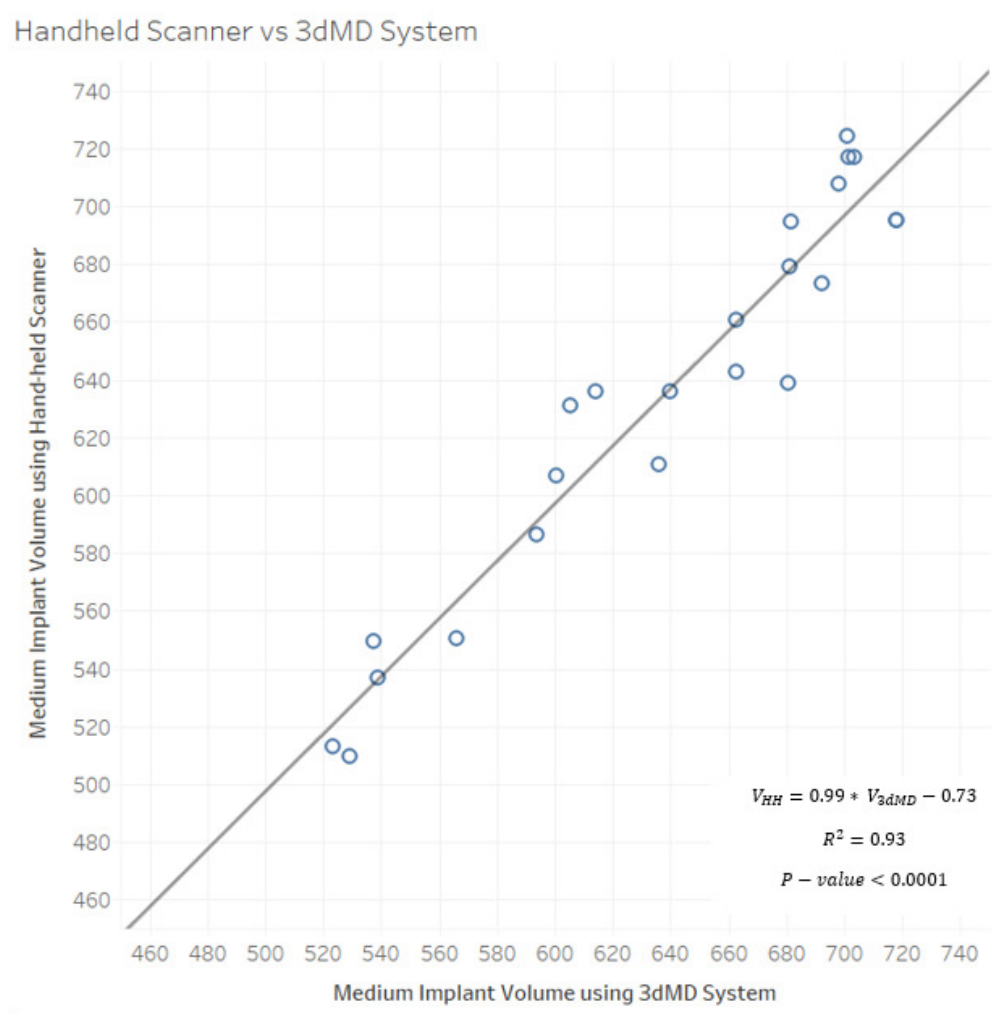

Fig. 7. Scatter plot of the measured volume of the images taken by the Go!SCAN $3 D^{T M}$ hand-held scanner vs the measured volume of the images taken by 3dMDtorso ${ }^{T M}$ system.

The calculated $R^{2}$ value was 0.93 with $p$-value $<0.001$ indicating correlation of volume calculated from images captured using $3 \mathrm{dMDtorso}{ }^{\mathrm{TM}}$ imaging system with the volume calculated from images captured using the Go!SCAN $3 \mathrm{D}^{\mathrm{TM}}$ hand-held scanner. This demonstrates the ability of the $3 \mathrm{D}$ hand-held scanner in capturing 3D surface images that enable quantitative measurements of volume similar to that of a previously validated stereophotography system [5].

\subsection{Investigation of the Amount of Volume Change Detectable using 3D Imaging}

In order to determine the change in volume detectable by 3D imaging, we utilized three different sized adjustable-volume breast implants described in the methods section. Volume adjustments were made in increments of $5 \mathrm{cc}$ and the implants (laid flat on a poster board) were imaged using the $3 \mathrm{dMDtorso} \mathrm{TM}^{\mathrm{TM}}$ system. As shown in Fig. 8, the volume of the implant was measured using our custom research software. The four landmark points for volume computation were annotated at the corners of the rectangle circumscribing the implant. Each volume measurement was performed 3 times and averaged to mitigate any random error occurring during the volume measurement process. In order to account for the volume of the empty (unfilled) implant, the empty implant volume was calculated using its weight and density. The measured volume was adjusted by subtracting the volume of the empty implant. Fig. 9 presents a plot of the measured volume versus the injected volume. To determine the smallest volume detectable, the volume difference was computed across each sequential image (5 cc change) and across every second sequential image (10 cc change) and was measured to be $5.51 \pm 2.95 \mathrm{cc}$ and $10.86 \pm 3.36 \mathrm{cc}$, respectively. The average error was $11.15 \pm 5.26 \mathrm{cc}$, amounting to $2.3 \pm 1.76 \%$ of the implant's volume (computed as (adjusted volume - injected volume) / (injected volume)). 

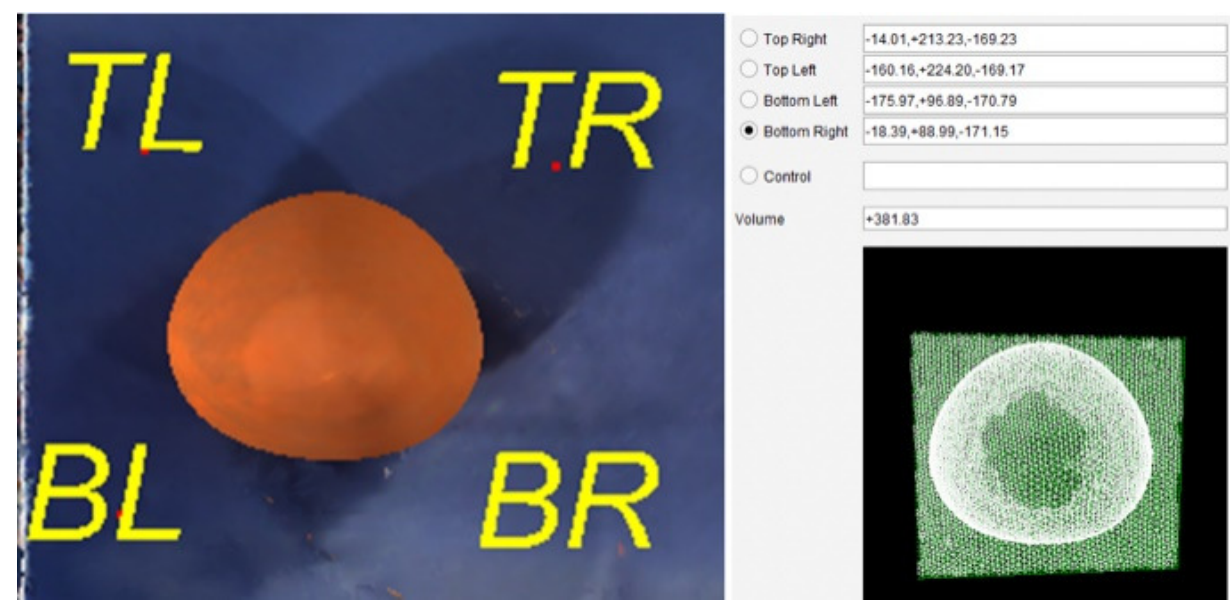

Fig. 8. Volume was measured as that enclosed between the implant surface and the Coons patch bound by points; TL: top left, TR: top right, BR; bottom right, and BL: bottom left.

As seen in Fig. 9, there is a linear correspondence between the measured volume and the injected volume $\left(R^{2}>0.99\right)$. This data suggests that $3 D$ imaging can successfully measure volume and can reliably detect changes in volume as low as $5 \mathrm{cc}$ for implants of varying sizes. A shown in Table 1 , Student's T-test indicated significance with $p$-values in the range of $10^{-4}-10^{-10}$ when comparing the 5 $\mathrm{cc}$ and $10 \mathrm{cc}$ volume difference measurements for each of the three sized implants.
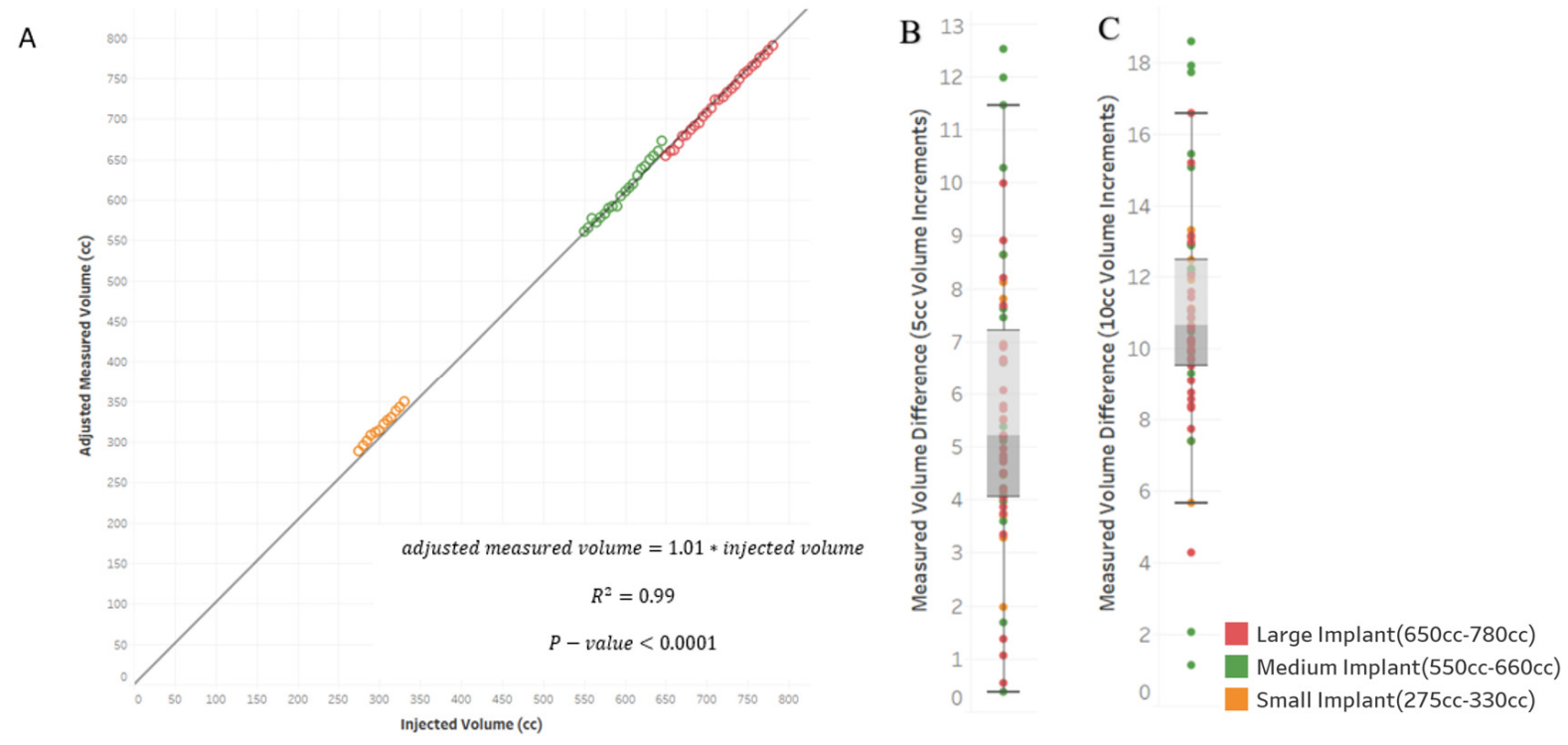

Fig. 9. Volume measurements in adjustable implants. (A) Measured volume versus injected volume, (B-C) Box plot of measured volume difference across incremental volume steps of $5 \mathrm{cc}$ and $10 \mathrm{cc}$.

Table 1. P-value of the Students t-test for two sample equal variance.

\section{Students T-Test}

Two sample equal variance

Two tail, $\alpha=0.05$

( 5 vs. 10 cc volume difference)

\begin{tabular}{|l|c|}
\hline \multicolumn{1}{|c|}{ Implant Size (cc) } & P-value \\
\hline $275-330$ & $6.5 \times 10^{-5}$ \\
\hline $550-660$ & $5.1 \times 10^{-4}$ \\
\hline $650-780$ & $2.9 \times 10^{-10}$ \\
\hline
\end{tabular}




\section{Conclusion}

In this study, we investigated the amount of volume change detectable using 3D imaging. We used adjustable implants to enable our investigation of small volume changes of $5 \mathrm{cc}$ and $10 \mathrm{cc}$. We also proposed a method to create a mannequin-based phantom to simulate the female torso, which incorporated the adjustable implants to afford the ability to manipulate breast volume in customizable increments/decrements. In addition, we also compared a commercially available and relatively inexpensive hand-held scanner with a sophisticated stereophotogrammetry system.

The proposed phantom was utilized to assess the resolution of 3D imaging for volume measurements as well as to investigate the applicability of using a 3D hand-held scanner in the clinic. While the phantom was effective in establishing the practicality of using the hand-held scanner for acquiring images of the female torso, and demonstrating its applicability for enabling breast volume measurements, its design was inappropriate for use in accurately monitoring small changes in breast volume. Using the $3 \mathrm{dMDtorso}{ }^{\mathrm{TM}}$ imaging system, the average error for volume computation for the medium size implant when placed on the phantom was found to be $32 \pm 18 \mathrm{cc}$ or $5.39 \pm 3.05 \%$. This value was $\sim 20 \mathrm{cc}$ higher than the error observed with computing the volume of the implants placed on the poster board which was $11.15 \pm 5.26 \mathrm{cc}$ or $2.3 \pm 1.76 \%$. This discrepancy was likely introduced by the design of the phantom. The injection of increasing amounts of liquid into the adjustable implants lifted the implant from the torso causing entrapment of air between the implant and the bodysuit. Using the adjustable implants on the poster board, we were able to measure volume differences of $5.5 \mathrm{cc} \pm$ $2.6 \mathrm{cc}$ for a pre-determined volume adjustment of $5 \mathrm{cc}$ to implant size, and volume differences of 10.86 $\pm 3.36 \mathrm{cc}$ for implant volume change of $10 \mathrm{cc}$.

Validation of the ability to use a hand-held scanner is essential as it gives the clinicians the ability to scan the patient's torso through $360^{\circ}$, compared to some configurations of $3 \mathrm{D}$ stereophotogrammetry systems that only capture the frontal surface through $180^{\circ}$ unless it is equipped with additional cameras positioned around the subject in order to image in $360^{\circ}$. Moreover, it enables the clinician (with minimal previous training) to image patients in the consulting room without having to move the patient to a dedicated room that is needed to house non-portable $3 \mathrm{D}$ imaging system, such as the $3 \mathrm{dMDtorso} \mathrm{TM}^{\mathrm{TM}}$ system. Likewise, the validation of the ability of 3D surface imaging to accurately detect small volume changes is important. For example, the ability to monitor small changes in volume can help in estimating fat retention volume in autologous fat grafting procedures that are frequently used for correcting contour defects in aesthetic and reconstructive breast surgery. Other applications include assessing breast volume changes that may occur over time during breast reconstruction, as well as in analyzing the volume asymmetry between breasts. Future work will focus on determining the smallest volume perceptible qualitatively by human observers.

\section{References}

[1] R. L. O'Connell, R. J. Stevens, P. A. Harris, and J. E. Rusby, "Review of three-dimensional (3D) surface imaging for oncoplastic, reconstructive and aesthetic breast surgery", The Breast, vol. 24, no. 4, pp. 331-342, 2015, https://doi.org/10.1016/j.breast.2015.03.011.

[2] C. Schmachtenberg, T. Fischer, B. Hamm, and U. Bick, "Diagnostic Performance of Automated Breast Volume Scanning (ABVS) Compared to Handheld Ultrasonography With Breast MRI as the Gold Standard", Academic Radiology, vol. 24, no. 8, pp. 954-961, 2017, https://doi.org/10.1016/j.acra.2017.01.021.

[3] J. Lee, M. Kawale, F. A. Merchant, J. Weston, M. C. Fingeret, D. Ladewig, G. P. Reece, M. A. Crosby, E. K. Beahm, and M. K. Markey, "Validation of Stereophotogrammetry of the Human Torso," Breast Cancer: Basic and Clinical Research, vol. 5, no. 1, pp 15-25, 2011, https://doi.org/10.4137/BCBCR.S6352.

[4] R. Kayar, S. Civelek, M. Cobanoglu, O. Gungor, H. Catal, and M. Emiroglu, "Five Methods of Breast Volume Measurement: A Comparative Study of Measurements of Specimen Volume in 30 Mastectomy Cases", Breast Cancer: Basic and Clinical Research, vol. 5, p. BCBCR.S6128, 2011, https://doi.org/10.4137/bcbcr.s6128.

[5] B. H. Howes, D. I. Watson, B. Fosh, J. M. Yip, P. Kleinig, and N. R. Dean, "Magnetic Resonance Imaging Versus 3-Dimensional Laser Scanning for Breast Volume Assessment After Breast Reconstruction", Annals of Plastic Surgery, vol. 78, no. 4, pp. 455-459, 2017, https://doi.org/10.1097/sap.0000000000000890. 
[6] F. H. K. Jeon, M. Griffin, A. Almadori, J. Varghese, S. Bogan, I. Younis, A. Mosahebi, and P. E. Butler, "Measuring Differential Volume Using the Subtraction Tool for Three-Dimensional Breast Volumetry: A Proof of Concept Study," Surgical Innovation, p. 155335062094556, 2020, https://doi.org/10.1177/1553350620945563.

[7] R. L. O'Connell, K. Khabra, J. C. Bamber, N. Desouza, F. Meybodi, P. A. Barry, and J. E. Rusby, "Validation of the Vectra XT three-dimensional imaging system for measuring breast volume and symmetry following oncological reconstruction," Breast Cancer Research and Treatment, vol. 171, no. 2, pp. 391-398, 2018, https://doi.org/10.1007/s10549-018-4843-6.

[8] T. S. Wesselius, A. C. Verhulst, R. D. Vreeken, T. Xi, T. J. J. Maal, and D. J. O. Ulrich, "Accuracy of Three Software Applications for Breast Volume Calculations from Three-Dimensional Surface Images," Plastic and Reconstructive Surgery, vol. 142, no. 4, pp. 858-865, 2018, https://doi.org/10.1097/PRS.0000000000004728.

[9] H. Henseler, A. Kuznetsova, P. Vogt, and B. Rosenhahn, "Validation of the Kinect device as a new portable imaging system for three-dimensional breast assessment," Journal of Plastic, Reconstructive \& Aesthetic Surgery, vol. 67, no. 4, pp. 483-488, 2014, https://doi.org/10.1016/j.bjps.2013.12.025.

[10] S. Choppin, J. Wheat, M. Gee, and A. Goyal, "The accuracy of breast volume measurement methods: A systematic review," The Breast, vol. 28, pp. 121-129, 2016, https://doi.org/10.1016/j.breast.2016.05.010.

[11] "[Hands-on] Test and review of the Creaform Go!SCAN 50, a professional-grade portable 3D scanner." [Online]. Available: https://www.aniwaa.com/blog/hands-on-test-and-review-of-thecreaform-goscan-50-portable-3d-scanner/ [Accessed: 22-June-2020].

[12] G. P. Reece, F. Merchant, J. Andon, H. Khatam, K. Ravi-Chandar, J. Weston, M. C. Fingeret, C. Lane, K. Duncan, and M. K. Markey, "3D surface imaging of the human female torso in upright to supine positions," Medical Engineering \& Physics, vol. 37, no. 4, pp. 375-383, 2015, https://doi.org/10.1016/..medengphy.2015.01.011. 